\title{
P01-109
}

\section{THE SOCIAL INTERVENTION FOR PEOPLE WITH ALZHEIMER DISEASE IN TRANSYLVANIA REGION}

\section{Stoica}

Alba University, Alba lulia, Romania

The Alzheimer disease affects mostly old people but sometimes even adults; it manifests itself as a series of brain modifications which determine several perturbations: thinking, memory, language, mood disturbances. All these disturbances take place gradually and strengthens as the illness advances. The exact causes that determine the Alzheimer disease are still unknown, that is why it can not be prevented or cured. Although, a diagnostic in the first stages and treatment as soon as possible is absolutely necessary for slowing it's evolution.

Unfortunately, the number of persons affected by Alzheimer is rising in Transylvania region. I estimated that only $12-15 \%$ of the cases are discovered in the early stages. The effect of the illness doesn't just affect the ill one, but all his family. Taking care of someone who suffers from Alzheimer means a lot of effort, because he needs permanently supervision and help in any kind of activity. Moreover, most of the times, the family is notable to take care of the sick person at home, and it has to take him to a residential center. Although, this is not always the best solution, especially that after being institutionalized, they are often having a nervous breakdown and the disease has a bad evolution. The social worker plays a very important role in helping the person who suffers from Alzheimer. The social intervention has to consider not only the specific needs of the ill, but also the family needs and it has to take place in both directions. 